\title{
Vitamin D and risk of pregnancy related hypertensive disorders: mendelian randomisation study
}

\author{
Maria C Magnus, ${ }^{1,2,3}$ Kozeta Miliku, ${ }^{4,5,6}$ Anna Bauer, ${ }^{7}$ Stephanie M Engel, ${ }^{7}$ Janine F Felix, ${ }^{4,5,6}$ \\ Vincent W V Jaddoe, ${ }^{4,5,6}$ Debbie A Lawlor, ${ }^{1,2,8}$ Stephanie J London, ${ }^{9}$ Per Magnus, ${ }^{3}$ \\ Ralph McGinnis, ${ }^{10}$ Wenche Nystad, ${ }^{11}$ Christian M Page, ${ }^{11,12}$ Fernando Rivadeneira, $, 4,6,13$ \\ Lars C Stene, ${ }^{11}$ German Tapia, ${ }^{11}$ Nicholas Williams, ${ }^{10}$ Carolina Bonilla, ${ }^{1,2,14}$ \\ Abigail Fraser ${ }^{1,2,8}$
}

For numbered affiliations see end of article.

Correspondence to: M C Magnus Maria.Christine. Magnus@brist

ol.ac.uk

Additional material is published online only. To view please visit the journal online.

Cite this as: BMJ 2018;361:k2167 http://dx.doi.org/10.1136/bmj.k2167

Accepted: 26 April 2018

\section{ABSTRACT}

OBJECTIVE

To use mendelian randomisation to investigate whether 25-hydroxyvitamin D concentration has a causal effect on gestational hypertension or preeclampsia.

DESIGN

One and two sample mendelian randomisation analyses.

SETTING

Two European pregnancy cohorts (Avon Longitudinal Study of Parents and Children, and Generation R Study), and two case-control studies (subgroup nested within the Norwegian Mother and Child Cohort Study, and the UK Genetics of Pre-eclampsia Study).

\section{PARTICIPANTS}

7389 women in a one sample mendelian randomisation analysis (751 with gestational hypertension and 135 with pre-eclampsia), and 3388 pre-eclampsia cases and 6059 controls in a two sample mendelian randomisation analysis.

EXPOSURES

Single nucleotide polymorphisms in genes associated with vitamin D synthesis (rs10741657 and rs12785878) and metabolism (rs6013897 and rs2282679) were used as instrumental variables.

\section{MAIN OUTCOME MEASURES}

Gestational hypertension and pre-eclampsia defined according to the International Society for the Study of Hypertension in Pregnancy.

\section{WHAT IS ALREADY KNOWN ON THIS TOPIC}

Observational studies find that women with lower levels of 25 -hydroxyvitamin D are at greater risk of pre-eclampsia

Some trials of vitamin D supplementation in pregnancy suggest a potential benefit of supplementation but they are small, heterogeneous in timing and dose, and have substantial attrition

Therefore, it is unclear whether vitamin $D$ is a cause of pre-eclampsia

\section{WHAT THIS STUDY ADDS}

A mendelian randomisation analysis of data from several European cohorts, showed no evidence to support a causal effect of 25 -hydroxyvitamin D levels on risk of pre-eclampsia or gestational hypertension

Further mendelian randomisation studies are needed with a larger number of women with pre-eclampsia, or which incorporate additional genetic instruments thereby increasing the proportion of 25 -hydroxyvitamin D explained

\section{RESULTS}

In the conventional multivariable analysis, the relative risk for pre-eclampsia was $1.03(95 \%$ confidence interval 1.00 to 1.07$)$ per $10 \%$ decrease in 25-hydroxyvitamin D level, and 2.04 (1.02 to 4.07) for 25 -hydroxyvitamin D levels $<25 \mathrm{nmol} / \mathrm{L}$ compared with $\geq 75 \mathrm{nmol} / \mathrm{L}$. No association was found for gestational hypertension. The one sample mendelian randomisation analysis using the total genetic risk score as an instrument did not provide strong evidence of a linear effect of 25-hydroxyvitamin $\mathrm{D}$ on the risk of gestational hypertension or preeclampsia: odds ratio 0.90 (95\% confidence interval 0.78 to 1.03$)$ and 1.19 (0.92 to 1.52$)$ per $10 \%$ decrease, respectively. The two sample mendelian randomisation estimate gave an odds ratio for preeclampsia of 0.98 (0.89 to 1.07 ) per $10 \%$ decrease in 25-hydroxyvitamin D level, an odds ratio of 0.96 ( 0.80 to 1.15$)$ per unit increase in the log(odds) of 25 -hydroxyvitamin D level $<75 \mathrm{nmol} / \mathrm{L}$, and an odds ratio of 0.93 ( 0.73 to 1.19 ) per unit increase in the $\log$ (odds) of 25 -hydroxyvitamin D levels $<50 \mathrm{nmol} / \mathrm{L}$.

\section{CONCLUSIONS}

No strong evidence was found to support a causal effect of vitamin D status on gestational hypertension or pre-eclampsia. Future mendelian randomisation studies with a larger number of women with preeclampsia or more genetic instruments that would increase the proportion of 25-hydroxyvitamin D levels explained by the instrument are needed.

\section{Introduction}

Low circulating 25-hydroxyvitamin D levels are common in pregnant women. ${ }^{1}$ The biologically active form of vitamin D, 1,25-dihydroxyvitamin D3 (calcitriol), can suppress renin biosynthesis and vascular smooth muscle cell proliferation, modulates macrophage activity and cytokine production, ${ }^{23}$ and regulates transcription of genes linked to placental invasion, normal implantation, and angiogenesis. ${ }^{4}$ Therefore, vitamin D status is a plausible causal factor in gestational hypertension and pre-eclampsia.

Meta-analyses of observational studies suggest an inverse association between 25-hydroxyvitamin D concentration and pre-eclampsia, but the potential for bias remains. ${ }^{56} \mathrm{~A}$ recent meta-analysis of three high quality randomised controlled trials that evaluated vitamin D supplementation during pregnancy found no strong evidence of a protective effect on gestational hypertension (pooled risk ratio 1.69, 95\% confidence 
interval 0.73 to 3.92$)$ or pre-eclampsia $(1.09,0.43$ to 2.76). ${ }^{7}$

Triangulating findings from studies that use different designs and analytical approaches increases confidence in findings. ${ }^{8}$ Mendelian randomisation analysis uses genetic variants as instrumental variables to examine whether an association is causal because the allocation of genes at conception is random at the population level and therefore independent of confounding factors. ${ }^{9} 10$ This approach examines the association of genetically determined levels of the exposure in relation to the outcomes of interest. A one sample mendelian randomisation analysis requires information on the genetic variants, exposure, and outcome from the same individual. A two sample mendelian randomisation analysis uses estimates of the association between the genetic variants and the exposure from one sample and the association between the genetic variants and the outcome from a second sample. One sample mendelian randomisation provides researchers with greater control of the analysis-for example, they can determine how to analyse the exposure (as a continuum, or in categories) and examine the association between the genetic instruments and measured confounders. Two sample mendelian randomisation takes advantage of published summary estimates from large scale genome wide association studies, which often results in greater statistical power. Genome wide association studies show robust associations of genetic variants located in genes that act in the pathway for vitamin D synthesis (CYP2R1 and DHCR7/NADSYN1) and metabolism (eg, CYP24A1 and GC) with 25-hydroxyvitamin D level. ${ }^{11} 12$ If the alleles associated with lower 25-hydroxyvitamin D concentrations are also associated with a greater risk of gestational hypertension or pre-eclampsia, this points towards a causal effect of lower 25-hydroxyvitamin D levels.

We investigated the causal effect of 25-hydroxyvitamin D level on pregnancy related hypertensive disorders using genetic variants that are associated with 25-hydroxyvitamin D levels as instrumental variables in a mendelian randomisation analysis.

\section{Methods}

Study population for multivariable regression and one sample mendelian randomisation analyses

Information on genetic variants, antenatal 25-hydroxyvitamin D levels, gestational hypertension, and pre-eclampsia were available in the Avon Longitudinal Study of Parents and Children (ALSPAC) ${ }^{13} 14$ and the Generation R Study ${ }^{15}$ (see the online supplementary material for more details). Written informed consent was obtained from all participants. We excluded women with multiple births, of non-European ethnicity, and with hypertension before pregnancy. Women of non-European ethnicity included those who were not of European ancestry based on self reported information or principal component analysis of their genome. A total of 4066 women from ALSPAC and 3323 women from Generation $\mathrm{R}$ were available for analysis (eFigs 1 and 2).

\section{Measurement of antenatal 25-hydroxyvitamin D levels}

Antenatal 25-hydroxyvitamin D levels were measured in serum (ALSPAC) or plasma (Generation R) using liquid chromatography-tandem mass spectrometry. 25-hydroxyvitamin D levels were natural log transformed and standardised according to calendar time of blood sampling by adding to the population mean each person's residual from a linear regression model, including the sine cosine function of calendar time of blood sampling as predictors. Additional information about the adjustment for season is available in the online supplementary material. For the conventional multivariable analysis, we evaluated 25-hydroxyvitamin D levels continuously (by 10\% decrease) and also categorised according to level based on recommendations of the Endocrine Society: less than $25 \mathrm{nmol} / \mathrm{L}, 25-49.9 \mathrm{nmol} / \mathrm{L}, 50-74.9 \mathrm{nmol} / \mathrm{L}$, and $75 \mathrm{nmol} / \mathrm{L}$ or higher. ${ }^{16}$ We did not have adequate power to test non-linear associations in the one sample mendelian randomisation analysis as there were only 751 women with gestational hypertension (592 in ALSPAC and 159 in Generation R) and 135 women with pre-eclampsia (77 in ALSPAC and 58 in Generation R) across the two cohorts.

\section{Study population for the two sample mendelian} randomisation analysis

We also conducted a two sample mendelian randomisation analysis of pre-eclampsia using estimates of the associations between the genetic instruments and 25-hydroxyvitamin D 1 levels, and the associations between the genetic instruments and preeclampsia from two independent samples. We used published estimates of the associations between the genetic instruments and 25-hydroxyvitamin D levels from 21 European cohorts $(n=42024) .{ }^{17}$ Estimates of the association between the genetic variants and pre-eclampsia were available from two case-control studies: a subgroup of individuals from a validation study of pre-eclampsia nested within the Norwegian Mother and Child Cohort Study (MoBa) ${ }^{18}$ (1513 preeclampsia cases and 971 healthy controls) and the UK Genetics of Pre-eclampsia study (GOPEC) ${ }^{19}$ (1875 pre-eclampsia cases and 5088 healthy controls). Additional information is available in the online supplementary material.

\section{Genetic instruments for the one and two sample mendelian randomisation analyses}

Our theoretical framework is shown in eFig 3 . Genetic instruments were four single nucleotide polymorphisms (SNPs) in genes associated with vitamin D synthesis (CYP2R1/rs10741657 and DHCR7/rs12785878) and metabolism (CYP24A1/ rs6013897 and $G C /$ rs2282679), which have been confirmed to be associated with 25-hydroxyvitamin D levels. ${ }^{11}{ }^{12}$ Information about the genotyping and 
quality control procedures is available in the online supplementary material. The genetic instruments were considered individually and combined into synthesis, metabolism, and total genetic risk scores. We created weighted genetic risk scores by weighting the instruments by the published effect estimates that we used for our two sample mendelian randomisation. ${ }^{17}$ We also did two separate two sample mendelian randomisation analyses of 25-hydroxyvitamin D levels less than $75 \mathrm{nmol} / \mathrm{L}$ (compared with $75 \mathrm{nmol} / \mathrm{L}$ or more) and less than $50 \mathrm{nmol} / \mathrm{L}$ (compared with 50 $\mathrm{nmol} / \mathrm{L}$ or more) for pre-eclampsia; we used published estimates of the association between three instruments (rs10741657, rs12785878, and rs2282679) with these 25 -hydroxyvitamin D thresholds. ${ }^{12}$ Associations between the genetic instruments and low levels of 25-hydroxyvitamin D - such as less than $25 \mathrm{nmol} / \mathrm{L}-$ have not been published. The four SNPs were in Hardy-Weinberg equilibrium $(P \geq 0.01)$ and none was in linkage disequilibrium $\left(\mathrm{R}^{2} \leq 0.01\right)$.

\section{Definition of gestational hypertension and pre- eclampsia}

All cohorts assessed pre-existing hypertension by self report. Routine blood pressure and proteinuria measurements from antenatal medical records were used to define gestational hypertension and pre-eclampsia. Gestational hypertension and preeclampsia were defined according to the criteria of the International Society for the Study of Hypertension in Pregnancy. ${ }^{20}$ Women without pre-existing hypertension were classified as having gestational hypertension if they had a systolic blood pressure $\geq 140 \mathrm{~mm} \mathrm{Hg}$ and/ or diastolic blood pressure $\geq 90 \mathrm{~mm} \mathrm{Hg}$ on at least two occasions first occurring after 20 gestational weeks. Pre-eclampsia was defined as gestational hypertension in combination with proteinuria $(\geq 0.3 \mathrm{~g} / \mathrm{d})$. For the multivariable analysis and the one sample mendelian randomisation analysis, the outcome was categorised as women without any hypertension either before or during pregnancy (reference group), gestational hypertension only, and pre-eclampsia.

\section{Covariates adjusted for in multivariable regression analysis}

Information on covariates was available for the multivariable analysis: age, parity $(0,1,2$, and $\geq 3$ births), prepregnancy body mass index $(<18.5,18.5-24.9,25-$ 29.9 , and $\geq 30 \mathrm{~kg} / \mathrm{m}^{2}$ ), educational level (low, medium, and high), smoking status during pregnancy (never, former/until pregnancy was known, and continued smoking), serum calcium concentration (nmol/L) (ALSPAC)/energy adjusted calcium intake (mg) (Generation $\mathrm{R}$ ), and gestational week of sample collection. We also extracted information on any intake of vitamin D supplements during pregnancy, defined as either a pure vitamin D supplement, a multivitamin, or cod liver oil.

\section{Statistical analysis}

We ran all analyses separately for each cohort and combined the results of each cohort using a random effects meta-analysis. The degree of heterogeneity between the cohorts was estimated using the $\mathrm{I}^{2}$ statistic.

We first estimated the multivariable adjusted associations of 25-hydroxyvitamin D level with gestational hypertension and pre-eclampsia using multinomial logistic regression in ALSPAC and Generation R. Coefficients were multiplied by $\ln (0.9)$ to reflect the association per $10 \%$ decrease in 25-hydroxyvitamin D level. ${ }^{21}$ Missing data on covariates ranged from $0 \%$ to $13 \%$ in the two cohorts (eTable 1). Multivariable imputation of missing covariate values was done with chained equations (ALSPAC), or according to the fully conditional specification method predictive mean matching (Generation R). Twenty datasets with imputed values were generated. Details of how these methods were applied and comparison of observed and imputed data are given in the online supplementary material and eTable 2, respectively.

We estimated the association between the genetic instruments and natural log transformed 25-hydroxyvitamin D values using linear regression. The exponentiated coefficients were multiplied by 100 (exp(coef)-1×100) to reflect the per cent change in 25-hydroxyvitamin $\mathrm{D}$ level per increase in copy number of the risk allele associated with lower 25-hydroxyvitamin D levels, ${ }^{21}$ and we report the $\mathrm{R}^{2}$ and $\mathrm{F}$ statistic as indicators of the strength of the instruments. Our assumption in the mendelian randomisation analyses is that genetic variants will not be associated with confounding factors, and we tested this for observed confounders.

The associations of the genetic instruments with gestational hypertension and pre-eclampsia were estimated by multinomial logistic regression analysis, adjusting for seven genomic principal components in ALSPAC. Generation $\mathrm{R}$ did not have information on genome wide genotypes to generate principal components. We also estimated the magnitude of the causal effect of 25-hydroxyvitamin D level on gestational hypertension and pre-eclampsia using a two step instrumental variable analysis. Firstly, we estimated the genetically predicted 25 -hydroxyvitamin $\mathrm{D}$ values from a linear regression, where $\log (25-$ hydroxyvitamin D) was the outcome and the genetic instrument the exposure. Secondly, we used these genetically predicted 25-hydroxyvitamin D levels as the exposure in a logistic regression model of the outcomes of interest, adjusting for seven principal components (ALSPAC only). The standard error of the coefficients from the logistic regression model were estimated using bootstrapping. We then compared the estimates obtained from the multivariable regression analysis with the estimates from the instrumental variable analysis using a non-parametric bootstrapping test. The multivariable and one sample mendelian randomisation analyses were done in Stata version 14.

We obtained estimates of the association between the genetic variants and pre-eclampsia for the two sample mendelian randomisation analysis using logistic regression, adjusting for the first five principal 
components. We then estimated the causal effect of 25-hydroxyvitamin D level on pre-eclampsia with each SNP as an instrument using the Wald ratio. We combined the estimates of the causal effects of each single SNP as an instrument using an inverse variance weighted method, which is a linear regression analysis through the mean 25-hydroxyvitamin D level and proportion with pre-eclampsia for each individual SNP. ${ }^{22}$ This method assumes that there are no alternative ways for the genetic instruments to influence the risk of gestational hypertension or preeclampsia other than through 25-hydroxyvitamin D (no horizontal pleiotropy). To assess this assumption, we estimated the causal effect using mendelian randomisation-Egger regression, which is a similar regression method that does not force the regression line through the intercept. ${ }^{23} \mathrm{~A}$ non-zero intercept value from mendelian randomisation-Egger regression is an indicator of possible horizontal pleiotropy, whereas the slope provides an unbiased estimate in the presence of pleiotropy with the assumption that the pleiotropic effects of the genetic instruments are uncorrelated with their associations with the exposure. ${ }^{23}$ We assessed heterogeneity in the estimated causal effect using the individual genetic instruments with the $\mathrm{Q}$ statistic. For the secondary analysis of the two 25-hydroxyvitamin D cut-off levels, the associations are the odds ratio for preeclampsia per unit increase in the $\log$ (odds) of having a 25-hydroxyvitamin D level below the cut-off. The two sample mendelian randomisation analysis was done in R 3.2.2 (R Foundation, www.R-project.org).

\section{Patient and public involvement}

No patients were involved in setting the research question or the outcome measures, nor were they involved in developing plans for recruitment, design, or implementation of the study. No patients were asked to advise on interpretation or writing up of results. There are no plans to disseminate the results of the research to study participants or the relevant patient community.

\section{Results}

Distribution of 25-hydroxyvitamin D and genetic instruments by background characteristics

Supplementary eTable 1 shows the characteristics of the 7389 women included in the one sample mendelian randomisation analysis. In the two cohorts, 751 women had gestational hypertension (592 in ALSPAC and 159 in Generation R) and 135 had pre-eclampsia (77 in ALSPAC and 58 in Generation R). The distribution of background characteristics was similar in the observed and imputed datasets (eTable 2). 25-hydroxyvitamin D level was positively associated with age (difference 1-2 years) and education (difference in proportion with high education 7-9\%) in both cohorts, and inversely associated with smoking (difference in proportion of women who smoked during pregnancy 13-20\%) and body mass index (difference in proportion with normal body mass index 6-38\%) (eTables 3 and 4). In contrast, the genetic instruments showed no strong evidence of associations with the confounding factors
(eTables 5 and 6). Notably, we did not observe strong evidence of an association between intake of vitamin D supplements and the genetic instruments.

\section{Association of 25-hydroxyvitamin D levels with gestational hypertension and pre-eclampsia: multivariable regression analysis}

We did not find strong evidence of any (linear or nonlinear) association between 25-hydroxyvitamin D level and gestational hypertension in the multivariable analysis(eTable 7). However, weobserved weakevidence of a linear association between 25-hydroxyvitamin D level and pre-eclampsia (pooled adjusted relative risk 1.03 (95\% confidence interval 1.00 to 1.07 ) per 10\% decrease; eTable 7). A 25-hydroxyvitamin D level less than $25 \mathrm{nmol} / \mathrm{L}$ was associated with a twofold increased risk of pre-eclampsia compared with levels of $75 \mathrm{nmol} / \mathrm{L}$ or more (pooled adjusted relative risk 2.04 (1.02 to 4.07); eTable 7).

\section{Strength of genetic variants as instruments for 25-hydroxyvitamin D level}

Supplementary eTable 8 shows the strength of the genetic instruments in predicting 25-hydroxyvitamin D level in ALSPAC and Generation R. The synthesis score explained $0.2 \%(\mathrm{~F}=9, \mathrm{n}=4062$ and $0.6 \%(\mathrm{~F}=21$, $\mathrm{n}=3275$ ) of the variation in 25-hydroxyvitamin D levels in ALSPAC and Generation R, respectively. In comparison, the metabolism score explained $1.2 \%(\mathrm{~F}=49$, $\mathrm{n}=3920)$ and $1.5 \%(\mathrm{~F}=52, \mathrm{n}=3284)$ of the variation in 25-hydroxyvitamin D levels in ALSPAC and Generation $\mathrm{R}$, respectively. For the published estimates of the associations of the genetic instruments and 25-hydroxyvitamin D level used for the two sample mendelian randomisation analysis, the synthesis score explained $0.6 \%$ of the variation in 25 -hydroxyvitamin D levels $(\mathrm{F}=230, \mathrm{n}=35$ 873), whereas the metabolism score explained $1.3 \%$ of the variation in 25 -hydroxyvitamin $\mathrm{D}$ levels $(\mathrm{F}=489, \mathrm{n}=38191){ }^{17}$

\section{Association of 25-hydroxyvitamin D level with} gestational hypertension and pre-eclampsia: one sample mendelian randomisation analysis

We found no consistent evidence of any associations of the three genetic risk scores with gestational hypertension or pre-eclampsia in the two cohorts (fig 1). When we evaluated the four SNPs individually, we found weak evidence of an association between a greater copy number of the 25-hydroxyvitamin D risk allele in rs2282679 and pre-eclampsia (eTable 9). The instrumental variable analysis also showed no consistent evidence of a causal linear effect of 25-hydroxyvitamin D level on the risk of gestational hypertension or pre-eclampsia: the odds ratio for gestational hypertension was 0.90 (95\% confidence interval 0.78 to 1.03 ) per $10 \%$ decrease in 25-hydroxyvitamin D level, and for preeclampsia was 1.19 (0.92 to 1.52) per $10 \%$ decrease in 25-hydroxyvitamin $\mathrm{D}$ level, when using the total genetic risk score as the instrument (fig 2 and eTable 10). When we compared the results from multivariable 


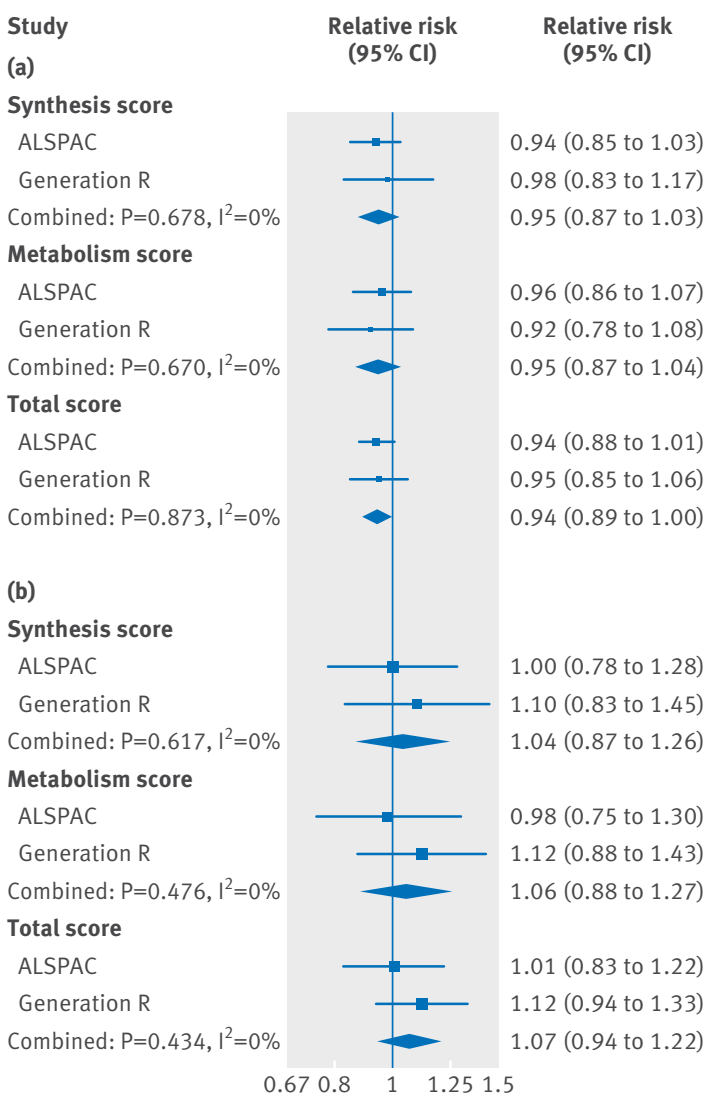

Fig 1 | Associations of the three genetic risk scores for 25-hydroxyvitamin D level with gestational hypertension and pre-eclampsia from the one sample mendelian randomisation analysis of the Avon Longitudinal Study of Parents and Children (ALSPAC) and the Generation R Study. (a) Gestational hypertension, (b) pre-eclampsia. Measures of association were obtained from multinomial logistic regression analysis. Associations reflect the additive risk of each additional copy of the risk allele associated with decreased 25-hydroxyvitamin D levels, and are adjusted for seven principal components to account for population stratification (ALSPAC only)

and instrumental variable analyses, no strong evidence was found that the estimates differed (in ALSPAC, $\mathrm{P}=0.17$ for gestational hypertension and 0.77 for preeclampsia; in Generation $\mathrm{R}, \mathrm{P}=0.10$ for gestational hypertension and 0.12 for pre-eclampsia). Based on a question raised in the review process, since rs6013897 was not a strong instrument for 25-hydroxyvitamin D levels, we conducted an analysis where we excluded rs6013897 from the total genetic risk score. This sensitivity analysis did not change our findings: the odds ratio for gestational hypertension was 0.91 (0.80 to 1.04 ) per $10 \%$ decrease in 25 -hydroxyvitamin D level, and for pre-eclampsia was 1.21 (0.94 to 1.57) per $10 \%$ decrease in 25-hydroxyvitamin D level.

\section{Association of 25-hydroxyvitamin D level with pre-eclampsia: two sample mendelian randomisation analysis}

Supplementary eTable11 shows the risk allele frequencies in the two-case control samples of

$\begin{array}{lcc}\text { Study } & \begin{array}{c}\text { Odds ratio } \\ (95 \% \mathrm{Cl})\end{array} & \begin{array}{c}\text { Odds ratio } \\ (\mathrm{a})\end{array} \\ & & 95 \% \mathrm{Cl})\end{array}$

Synthesis score

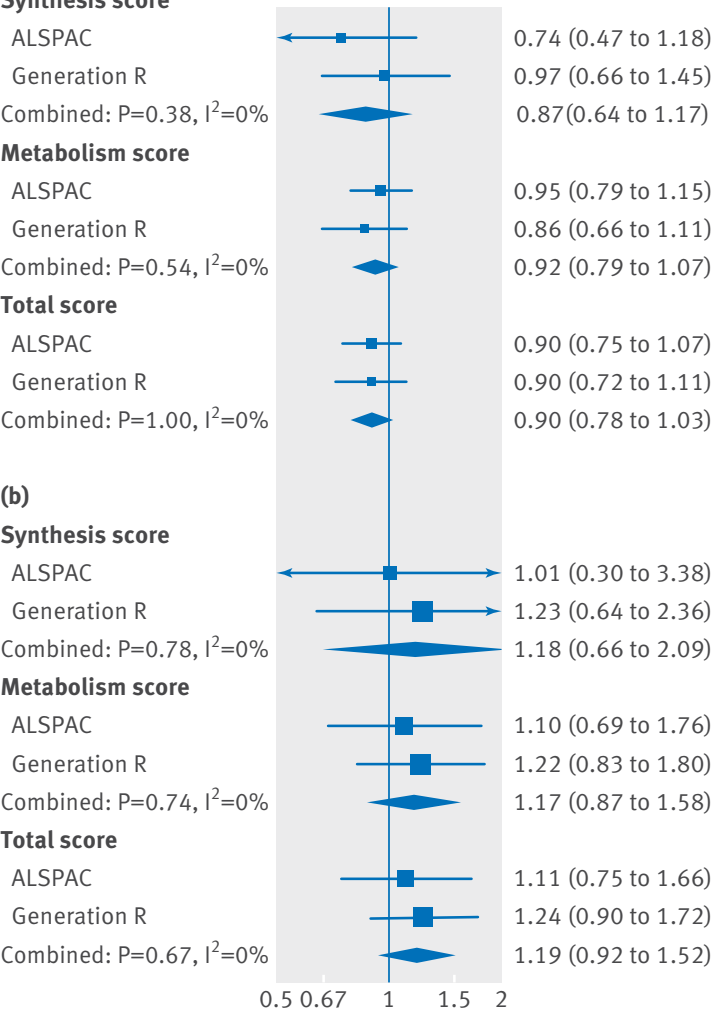

Fig 2 | Causal associations of 25 -hydroxyvitamin D level with gestational hypertension and pre-eclampsia from the one sample mendelian randomisation analysis of the Avon Longitudinal Study of Parents and Children (ALSPAC) and the Generation R Study. (a) Gestational hypertension, (b) pre-eclampsia. The causal association was estimated using two step instrumental variable analysis, and associations reflect the change in risk per $10 \%$ decrease in 25 -hydroxyvitamin D levels. The associations are adjusted for gestational week of blood sampling and seven principal components to account for population stratification (ALSPAC only)

pre-eclampsia (MoBa and GOPEC). Similar to the one sample mendelian randomisation analysis, we found no associations between the genetic instruments for 25-hydroxyvitamin D levels and pre-eclampsia in the two sample mendelian randomisation analysis (fig 3 and eTable 12), and no evidence of a causal linear effect in the formal two sample mendelian randomisation analysis, (odds ratio 0.98 (95\% confidence interval 0.89 to 1.07 ) per $10 \%$ decrease in 25 -hydroxyvitamin $\mathrm{D}$ level (fig 4 and eTable 13). The $\mathrm{P}$ values from the $\mathrm{Q}$ statistic were 0.58 in GOPEC and 0.89 in $\mathrm{MoBa}$ (inverse variance weighted method), indicating no evidence of heterogeneity in the estimates of the genetic instruments. Furthermore, we found no strong evidence of horizontal pleiotropy: mendelian randomisation-Egger intercept $-0.06(\mathrm{P}=0.34)$ in GOPEC and $0.04(\mathrm{P}=0.56)$ in MoBa. Similar to what we observed in the one sample mendelian randomisation analysis, excluding rs6013897 from the total genetic risk score did not change our findings, odds ratio 


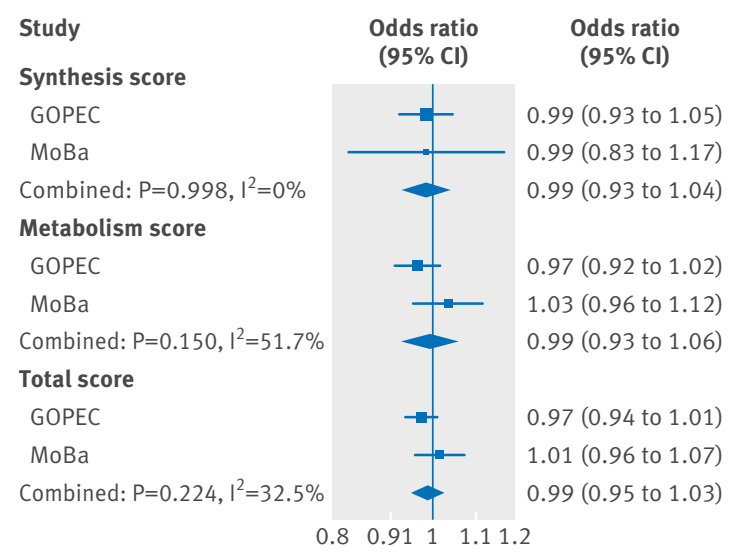

Fig 3 | Associations of the three genetic risk scores for 25 -hydroxyvitamin D level with pre-eclampsia from the two sample mendelian randomisation analysis of the UK Genetics of Pre-eclampsia Study (GOPEC) and the Norwegian Mother and Child Cohort Study (MoBa). The associations were estimated by ordinary logistic regression analysis, and reflect the additive risk of each additional copy of the risk allele associated with decreased 25-hydroxyvitamin $D$ levels. The estimates are adjusted for five principal components

0.97 (0.88 to 1.08$)$ per $10 \%$ decrease in 25 -hydroxyvitamin D levels. In the two sample mendelian randomisation analysis of 25-hydroxyvitamin D cutoff levels, no strong evidence was found of a causal effect of 25-hydroxyvitamin D level on the risk of pre-eclampsia: the odds ratio for pre-eclampsia was 0.96 (0.80 to 1.15 ) per unit increase in the $\log$ (odds) of having a 25-hydroxyvitamin D level less than 75 $\mathrm{nmol} / \mathrm{L}$ and 0.93 (0.73 to 1.19) per unit increase in the

\begin{tabular}{|c|c|c|}
\hline Study & $\begin{array}{c}\text { Odds ratio } \\
(95 \% \mathrm{Cl})\end{array}$ & $\begin{array}{c}\text { Odds ratio } \\
(95 \% \mathrm{Cl})\end{array}$ \\
\hline \multicolumn{3}{|l|}{ Synthesis score } \\
\hline GOPEC & & $0.96(0.80$ to 1.15$)$ \\
\hline $\mathrm{MoBa}$ & & $0.96(0.75$ to 1.22$)$ \\
\hline Combined: $P=0.998, I^{2}=0 \%$ & & $0.96(0.83$ to 1.11$)$ \\
\hline \multicolumn{3}{|l|}{ Metabolism score } \\
\hline GOPEC & & 0.94 (0.81 to 1.08$)$ \\
\hline $\mathrm{MoBa}$ & & $1.07(0.92$ to 1.24$)$ \\
\hline Combined: $P=0.207,1^{2}=37.3 \%$ & & $1.00(0.88$ to 1.13$)$ \\
\hline \multicolumn{3}{|l|}{ All (inverse variance weighted) } \\
\hline GOPEC & & 0.94 (0.86 to 1.03$)$ \\
\hline MoBa & & $1.04(0.91$ to 1.18$)$ \\
\hline Combined: $P=0.223, I^{2}=32.7 \%$ & & 0.98 (0.89 to 1.07$)$ \\
\hline \multicolumn{3}{|l|}{ All (MR Egger) } \\
\hline GOPEC & & 0.85 (0.71 to 1.02$)$ \\
\hline MoBa & & $1.12(0.87$ to 1.46$)$ \\
\hline Combined: $P=0.086, I^{2}=66.1 \%$ & & $0.96(0.74$ to 1.26$)$ \\
\hline
\end{tabular}

Fig 4 | Causal association between 25-hydroxyvitamin D level and pre-eclampsia from the two sample mendelian randomisation (MR) analysis of the UK Genetics of Preeclampsia Study (GOPEC) and the Norwegian Mother and Child Cohort Study (MoBa). Associations are estimated using the inverse variance weighted and MR-Egger methods, and reflect the change in risk per $10 \%$ decrease in 25-hydroxyvitamin D levels

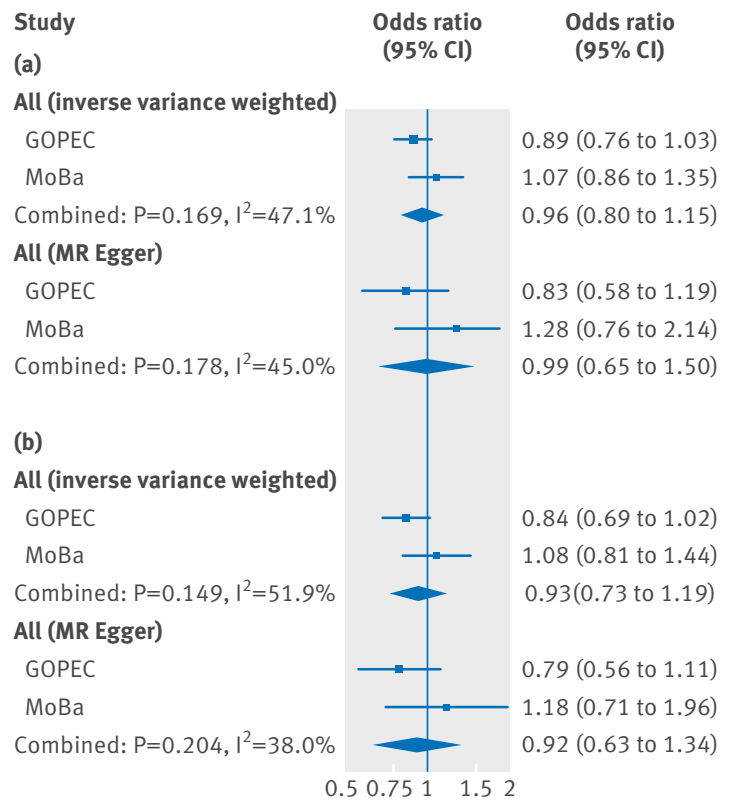

Fig 5 | Causal association between 25-hydroxyvitamin D cut-off levels and pre-eclampsia from the two sample mendelian randomisation (MR) analysis of the UK Genetics of Pre-eclampsia Study (GOPEC) and the Norwegian Mother and Child Cohort Study (MoBa). (a) $<75 \mathrm{nmol} / \mathrm{L}$ compared with $\geq 75 \mathrm{nmol} / \mathrm{L}$, (b) $<50 \mathrm{nmol} / \mathrm{L}$ compared with $\geq 50 \mathrm{nmol} / \mathrm{L}$. Associations reflect the risk of pre-eclampsia per increase in the $\log (\mathrm{odds})$ of the risk of being below the respective cut-off level for 25-hydroxyvitamin D

$\log$ (odds) of having a 25-hydroxyvitamin D level less than $50 \mathrm{nmol} / \mathrm{L}$ (fig 5 and eTable 14).

\section{Discussion}

A lower 25-hydroxyvitamin D level was weakly associated with a lower risk of gestational hypertension and higher risk of pre-eclampsia in the one sample mendelian randomisation analysis. This could indicate that the null association between 25 -hydroxyvitamin $\mathrm{D}$ level and gestational hypertension in the multivariable analysis was influenced by unobserved confounding. Further evidence from larger studies is needed to provide conclusive evidence. Furthermore, no evidence was found of an association between 25-hydroxyvitamin D level and pre-eclampsia in the larger two sample mendelian randomisation. Nor did we find any appreciable evidence of a causal effect of 25 -hydroxyvitamin D levels less than $75 \mathrm{nmol} / \mathrm{L}$ or less than $50 \mathrm{nmol} / \mathrm{L}$ on the risk of pre-eclampsia.

\section{Strengths and limitations of this study}

The main strengths of our study are the use of genetic variants as instrumental variables to reduce the possibility of confounding, and our inclusion of multiple cohorts. The methods for ascertaining outcome were similar for the cohorts but we acknowledge a difference in the proportion of women with gestational hypertension in ALSPAC (15\%) and Generation R (5\%) cohorts. This is likely explained by the fact that 
Generation $\mathrm{R}$ relied on registered diagnoses in the antenatal charts, whereas ALSPAC abstracted blood pressure and proteinuria measures from the charts and then defined the outcomes according to the guidelines. We accounted for some observed heterogeneity in the associations between the cohorts, which might be explained by the difference in ascertaining the outcome, by running a random effects meta-analysis instead of a fixed effects meta-analysis. Mendelian randomisation analysis has limitations. Horizontal pleiotropy could bias our findings. We observed no consistent evidence of associations between the genetic instruments and a range of background characteristics in the two pregnancy cohorts included in the one sample mendelian randomisation analysis. This might be explained by the modest sample size. However, a study of 6877 Europeans in the 1958 British Birth Cohort reported no associations between the four genetic instruments that we used in the current study and a wide range of potential pleiotropic pathways, including oily fish consumption, smoking, body mass index, abdominal obesity, social class, and C reactive protein levels. ${ }^{24}$ Therefore, no strong evidence exists of pleiotropy for the genetic instruments for 25-hydroxyvitamin D. This is further supported by the findings of the mendelian randomisation-Egger regression that had an intercept of $-0.06(\mathrm{P}=0.34)$ in GOPEC and $0.04(\mathrm{P}=0.56)$ in MoBa. ${ }^{23}$ Our analyses were restricted to pregnant women. If vitamin D status affects fertility, this might have resulted in selection bias. ${ }^{25} 26$

We explored the association between the genetic instruments and intake of vitamin D supplements because if women with lower genetically predicted 25-hydroxyvitamin D levels are more likely to take supplements, this could theoretically distort our findings. The proportion of women who took vitamin D supplements during pregnancy differed in the two cohorts, which might reflect cultural, socioeconomic, or policy differences. However, we did not see strong evidence of an association between the genetic instruments and intake of vitamin D supplements in ALSPAC or Generation R. In addition, associations between the genetic instruments and measured 25-hydroxyvitamin D levels were similar to previous reports.

Finally, in the two sample mendelian randomisation analysis, we used estimates for the associations of the genetic instruments with 25-hydroxyvitamin D levels and pre-eclampsia from the largest samples available. Our two sample mendelian randomisation analysis of pre-eclampsia (3388 cases and 6059 controls), using the total genetic risk score as an instrument (explaining about $2 \%$ of the variation in 25 -hydroxyvitamin D levels), was adequately powered (80\%) to detect an odds ratio of 1.5 per standard deviation decrease in 25-hydroxyvitamin D level. ${ }^{27}$ Translating this to the association we were able to detect for a $10 \%$ decrease in 25-hydroxyvitamin D level (0.105 on the log scale), using the estimate of the standard deviation of natural log transformed 25-hydroxyvitamin D level in ALSPAC (0.45), this is equivalent to an odds of 1.10 per 10\% decrease in 25-hydroxyvitamin D level (exp $((\ln (1.5) / 0.45) \times 0.105)$. We therefore acknowledge that a more modest effect might be present that we were not able to detect. Future mendelian randomisation studies with a larger number of women with pre-eclampsia are therefore needed.

\section{Comparison with other studies}

Previous observational studies of 25-hydroxyvitamin D level and risk of pre-eclampsia report conflicting findings, as summarised in meta-analyses, ${ }^{5}{ }^{6}$ which report a pooled odds ratio for pre-eclampsia of 1.70 (95\% confidence interval 1.25 to 2.58 ) when comparing women with 25-hydroxyvitamin D levels less than $75 \mathrm{nmol} / \mathrm{L}$ with those with levels of 75 nmol/L or more. ${ }^{5}$ Estimates of the associations vary according to study design, whether the original studies had adjusted for important confounders, the categorisation of 25-hydroxyvitamin D, and 25-hydroxyvitamin D quantification method. Previous studies of 25-hydroxyvitamin D level and gestational hypertension also reported conflicting findings. ${ }^{28-31}$

Our findings are more in line with a recent systematic review and meta-analysis of randomised controlled trials of vitamin D supplementation in pregnancy. The pooled risk ratio of pre-eclampsia was 1.09 (95\% confidence interval 0.43 to 2.76 ), with a high degree of heterogeneity in the effects estimates between the three included trials $\left(\mathrm{I}^{2}=67 \%\right) .^{7}$ The sizes of the trials were modest, ranging between about 70 and 200 women in each study arm. ${ }^{32-34}$ The intervention also varied between the three studies, with 50000 IU $(1250 \mu \mathrm{g})$ of vitamin D3 given once every two weeks in two trials and $4000(100 \mu \mathrm{g})$ IU vitamin D3 given daily in one trial. ${ }^{32-34}$ The authors of the metaanalysis attribute the difference between their pooled estimate and the results of previous meta-analyses to their stricter exclusion criteria based on trial quality. ${ }^{7}$ We found four more ongoing trials looking at vitamin D supplementation and pre-eclampsia registered on ClinicalTrials.gov. The largest one aims to recruit a total of 460 women across the intervention arms.

Genetic predisposition to higher 25-hydroxyvitamin D levels and supplementation are not equivalent. ${ }^{35}$ While a randomised controlled trial is done at a given time point or period, mendelian randomisation estimates the effect of a lifelong difference in levels of the exposure. Mendelian randomisation might therefore not be the best approach if the association between the genetic instruments and the exposure of interest varies over the life course, or if there is a critical period for an effect of the exposure on the outcome of interest. ${ }^{8}$ The pathogenesis of pre-eclampsia likely starts during early pregnancy $^{36}$ and therefore women's nutrient status before pregnancy or at conception and during the first trimester may be of particular relevance. However, we are unaware of any evidence to support the notion that the association between the genetic instruments and 25-hydroxyvitamin D level varies greatly over the life course. Furthermore, genetically determined changes in exposure levels are usually small and assumed to be uniform in the population, whereas interventions 
assessed in randomised controlled trials have greater effects. For all of these reasons, extrapolating findings from a mendelian randomisation analysis beyond the modest genetic effect might not be valid. ${ }^{35}$

\section{Policy implications}

Debate continues about the recommendations for vitamin D intake in pregnancy. ${ }^{37}$ The US Institute of Medicine has set a recommended dietary allowance for vitamin $\mathrm{D}$ for pregnant and lactating women of 600 IU $(15 \mu \mathrm{g})$ daily, ${ }^{38}$ while the UK National Health Service recommends that all adults (including pregnant women) consume about $400 \mathrm{IU}(10 \mu \mathrm{g})$ each day, ${ }^{39}$ and the Health Council of the Netherlands suggests that all pregnant women take $400 \mathrm{IU}(10 \mu \mathrm{g})$ of vitamin D daily. In Norway, the health directorate recommends that pregnant women take one tablespoon of cod liver oil each day, which also contains $400 \mathrm{IU}(10 \mu \mathrm{g})$ of vitamin D. ${ }^{40}$ According to the World Health Organization, evidence recommending vitamin D supplementation for women during pregnancy to reduce adverse pregnancy outcomes is insufficient. ${ }^{41}$ Our findings support the current WHO position. Mendelian randomisation studies with a greater number of women with preeclampsia will provide additional evidence to support or negate a causal effect of vitamin D in pre-eclampsia. The next step (should such an effect be likely) would require large, well conducted trials of vitamin $\mathrm{D}$ supplementation. In particular, it is important to find out whether the benefit of vitamin D supplementation varies by ethnic group-most studies to date have included only individuals of European origin-and a broad range of baseline 25-hydroxyvitamin D levels.

\section{Conclusion}

Mendelian randomisation analyses using the largest available sample yielded no strong evidence to support a causal effect of vitamin D status on gestational hypertension or pre-eclampsia. Further mendelian randomisation studies are needed with a larger number of women with pre-eclampsia, or which incorporate additional genetic instruments thereby increasing the proportion of 25-hydroxyvitamin D explained. In combination with adequately powered clinical trials, this could help finally establish whether vitamin D status has a role in pregnancy related hypertensive disorders.

\section{AUTHOR AFFILIATIONS}

${ }^{1}$ Medical Research Council Integrative Epidemiology Unit, University of Bristol, Bristol BS8 2BN, UK

${ }^{2}$ Department of Population Health Sciences, Bristol Medical School, Bristol, UK

${ }^{3}$ Centre for Fertility and Health, Norwegian Institute of Public Health, Oslo, Norway

${ }^{4}$ Generation R Study Group, Erasmus MC, University Medical Centre Rotterdam, Rotterdam, Netherlands

${ }^{5}$ Department of Pediatrics, Erasmus MC, University Medical Centre Rotterdam, Rotterdam, Netherlands

${ }^{6}$ Department of Epidemiology, Erasmus MC, University Medical Centre Rotterdam, Rotterdam, Netherlands

${ }^{7}$ Gillings School of Global Public Health, University of North Carolina, Chapel Hill, NC, USA
${ }^{8}$ National Institute for Health Research Bristol Biomedical Research Centre, University Hospitals Bristol NHS Foundation Trust, and the University of Bristol, Bristol, UK

${ }^{9}$ Epidemiology Branch, National Institute of Environmental Health Sciences, National Institutes of Health, Department of Health and Human Services, Research Triangle Park, NC, USA

${ }^{10}$ Sanger Institute, University of Cambridge, Cambridge, UK

${ }^{11}$ Division for Mental and Physical Health, Norwegian Institute of Public Health, Oslo, Norway

${ }^{12}$ Oslo Centre for Biostatistics and Epidemiology, Oslo University Hospital, Oslo, Norway

${ }^{13}$ Department of Internal Medicine, Erasmus MC, University Medical Centre Rotterdam, Rotterdam, Netherlands

${ }^{14}$ Departamento de Medicina Preventiva, Faculdade de Medicina, Universidade de São Paulo, São Paulo, Brazil

We thank Jack Bowden at the MRC Integrative Epidemiology Unit (University of Bristol) for input on the statistical analysis; the participants in the Avon Longitudinal Study of Parents and Children (ALSPAC), Generation R Study, Norwegian Mother and Child Cohort Study (MoBa), and UK Genetics of Pre-eclampsia study (GOPEC); and those who contributed to the recruitment and the infrastructure surrounding the cohorts.

Contributors: AF, WN, LCS, SE, and VWVJ conceived and designed the study. RM obtained access to data from the different cohorts. All authors contributed to the design of the study. MCM, KM, NW, and $A B$ contributed to the data analysis. MCM drafted the manuscript. $\mathrm{CB}$ and $\mathrm{AF}$ are equal contributing senior authors. All authors had full access to all of the data (including statistical reports and tables) in the study and can take responsibility for the integrity of the data and the accuracy of the data analysis. All authors interpreted data and critically revised and approved the final manuscript. MCM is guarantor. The corresponding author attests that all listed authors meet authorship criteria and that no others meeting the criteria have been omitted.

Funding: This project received funding from the European Union's Horizon 2020 research and innovation programme under grant agreement No 633595 (DynaHEALTH), and the European Union's horizon 2020 research and innovation programme under grant agreement 733206 (LifeCycle Project). This work was also partly supported by the Research Council of Norway through its Centres of Excellence funding scheme, project number 262700.

Competing interests: All authors have completed the ICMJE uniform disclosure form at www.icmje.org/coi_disclosure.pdf (available on request from the corresponding author) and have the following declarations. MCM, AF, CB and DAL work at the Medical Research Council Integrative Epidemiology Unit at the University of Bristol which receives infrastructure funding from the UK MRC (MC/UU/12013/5). The contribution of AF and DAL were supported by the National Institute for Health Research Biomedical Research Centre at University Hospitals Bristol National Health Service Foundation Trust and the University of Bristol. MCM and AF are also supported by a UK MRC fellowship (MR/M009351/1) awarded to AF. DAL is also supported by the US National Institutes of Health (NF-SI-0611-10196) and a European Research Council Advanced Grant (DevelopObese; 669545); DAL is an NIHR Senior Investigator (R01 DK10324). DAL also reports funding from Roche Diagnostics and Medtronic for biomarker research, outside the current work. KM is supported through Erasmus Mundus Western Balkans (ERAWEB), a project funded by the European Commission. VWVJ received a grant from the Netherlands Organisation for Health Research and Development (NWO, ZonMw-VIDI 016.136.361) and a European Research Council Consolidator Grant (ERC-2014-CoG-648916). AEB was supported in part by the NIH (NICHD T32 HD052468). SIL is supported by the Intramural Research Program of the NIH (NIEHS). AB and SE report that genotyping of one of the cohorts included in the study was financed by the US NIH. JF reports a research grant from the European Research Council's Horizon 2020 scheme. The views expressed in this publication are those of the authors and not necessarily those of any of the funders or of the UK NHS or the Department of Health.

Ethical approval: The different cohorts had approvals from respective national ethical committees for medical research. Ethical approval for the ALSPAC study was granted by the ALSPAC Law and Ethics Committee and the local research ethics committees. The data collection and analysis of MoBa were approved by the Norwegian Data Inspectorate and the regional committees for medical and health research ethics. The Generation R Study was approved by the local 
medical ethical committee of the Erasmus Medical Centre, Rotterdam. GOPEC was approved by the Trent Multicentre Research Ethics Committee, United Kingdom.

Data sharing: No additional data available.

Transparency: The lead author (MCM) affirms that the manuscript is an honest, accurate, and transparent account of the study being reported; that no important aspects of the study have been omitted; and that any discrepancies from the study as planned have been explained.

This is an Open Access article distributed in accordance with the terms of the Creative Commons Attribution (CC BY 4.0) license, which permits others to distribute, remix, adapt and build upon this work, for commercial use, provided the original work is properly cited. See: http://creativecommons.org/licenses/by/4.0/.

1 Palacios C, Gonzalez L. Is vitamin D deficiency a major global public health problem? J Steroid Biochem Mol Biol 2014;144(Pt A):138-45. doi:10.1016/j.jsbmb.2013.11.003

2 Cardús A, Parisi E, Gallego C, Aldea M, Fernández E, Valdivielso IM. 1,25-Dihydroxyvitamin D3 stimulates vascular smooth muscle cell proliferation through a VEGF-mediated pathway. Kidney Int 2006;69:1377-84. doi:10.1038/sj.ki.5000304

3 Li YC, Kong J, Wei M, Chen ZF, Liu SQ, Cao LP. 1,25-Dihydroxyvitamin $D(3)$ is a negative endocrine regulator of the renin-angiotensin system. J Clin Invest 2002;110:229-38. doi:10.1172/JCI0215219

4 Evans KN, Bulmer JN, Kilby MD, Hewison M. Vitamin D and placentaldecidual function. J Soc Gynecol Investig 2004:11:263-71. doi:10.1016/j.jsgi.2004.02.002

5 Aghajafari F, Nagulesapillai T, Ronksley PE, Tough SC, O’Beirne M, Rabi DM. Association between maternal serum 25-hydroxyvitamin $D$ level and pregnancy and neonatal outcomes: systematic review and meta-analysis of observational studies. BMJ 2013;346:f1169. doi:10.1136/bmj.f1169

6 Purswani JM, Gala P, Dwarkanath P, Larkin HM, Kurpad A, Mehta S. The role of vitamin $D$ in pre-eclampsia: a systematic review. BMC Pregnancy Childbirth 2017;17:231. doi:10.1186/s12884-0171408-3

7 Roth DE, Leung M, Mesfin E, Qamar H, Watterworth J, Papp E. Vitamin D supplementation during pregnancy: state of the evidence from a systematic review of randomised trials. BMJ 2017;359:15237. doi:10.1136/bmj.j5237

8 Lawlor DA, Tilling K, Davey Smith G. Triangulation in aetiological epidemiology. Int J Epidemiol 2016;45:1866-86. doi:10.1093/ije/ dyw314

9 Lawlor DA, Harbord RM, Sterne JA, Timpson N, Davey Smith G. Mendelian randomization: using genes as instruments for making causal inferences in epidemiology. Stat Med 2008;27:1133-63. doi:10.1002/sim.3034

10 Smith GD, Ebrahim S. 'Mendelian randomization': can genetic epidemiology contribute to understanding environmental determinants of disease? Int J Epidemiol 2003;32:1-22. doi:10.1093/ije/dyg070

11 Ahn J, Yu K, Stolzenberg-Solomon R, et al. Genome-wide association study of circulating vitamin D levels. Hum Mol Genet 2010;19:2739 45. doi:10.1093/hmg/ddq155

12 Wang TJ, Zhang F, Richards JB, et al. Common genetic determinants of vitamin D insufficiency: a genome-wide association study. Lancet 2010;376:180-8. doi:10.1016/S0140-6736(10)60588-0

13 Fraser A, Macdonald-Wallis C, Tilling K, et al. Cohort Profile: the Avon Longitudinal Study of Parents and Children: ALSPAC mothers cohort. Int J Epidemiol 2013;42:97-110. doi:10.1093/ije/dys066

14 Boyd A, Golding J, Macleod J, et al. Cohort Profile: the "children of the 90s'--the index offspring of the Avon Longitudinal Study of Parents and Children. Int J Epidemiol 2013;42:111-27. doi:10.1093/ije/ dys064

15 Kooijman MN, Kruithof CJ, van Duijn CM, et al. The Generation R Study: design and cohort update 2017. Eur Epidemiol 2016;31:1243-64. doi:10.1007/s10654-016-0224-9

16 Holick MF, Binkley NC, Bischoff-Ferrari HA, et al, Endocrine Society. Evaluation, treatment, and prevention of vitamin D deficiency: an Endocrine Society clinical practice guideline. J Clin Endocrinol Metab 2011;96:1911-30. doi:10.1210/jc.2011-0385

17 Vimaleswaran KS, Berry DJ, Lu C, et al, Genetic Investigation of Anthropometric Traits-GIANT Consortium. Causal relationship between obesity and vitamin D status: bi-directional Mendelian randomization analysis of multiple cohorts. PLOS Med 2013;10:e1001383. doi:10.1371/journal.pmed.1001383

18 Klungsøyr K, Harmon QE, Skard LB, et al. Validity of pre-eclampsia registration in the medical birth registry of Norway for women participating in the Norwegian mother and child cohort study, 19992010. Paediatr Perinat Epidemiol 2014;28:362-71. doi:10.1111/ ppe.12138
19 GOPEC Consortium. Disentangling fetal and maternal susceptibility for pre-eclampsia: a British multicenter candidate-gene study. Am Hum Genet 2005;77:127-31. doi:10.1086/431245

20 Brown MA, Lindheimer MD, de Swiet M, Van Assche A, Moutquin IM. The classification and diagnosis of the hypertensive disorders of pregnancy: statement from the International Society for the Study of Hypertension in Pregnancy (ISSHP). Hypertens Pregnancy 2001;20:IX-XIV. doi:10.3109/10641950109152635

21 Cole TJ. Sympercents: symmetric percentage differences on the $100 \log (\mathrm{e})$ scale simplify the presentation of log transformed data. Stat Med 2000;19:3109-25. doi:10.1002/10970258(20001130)19:22<3109:.AID-SIM558>3.0.CO·2-F

22 Burgess S, Butterworth A, Thompson SG. Mendelian randomization analysis with multiple genetic variants using summarized data. Genet Epidemiol 2013;37:658-65. doi:10.1002/gepi.21758

23 Bowden J, Davey Smith G, Burgess S. Mendelian randomization with invalid instruments: effect estimation and bias detection through Egge regression. Int J Epidemiol 2015;44:512-25. doi:10.1093/ije/dyv080

24 Berry DJ, Vimaleswaran KS, Whittaker JC, Hingorani AD, Hyppönen E. Evaluation of genetic markers as instruments for Mendelian randomization studies on vitamin D. PLoS One 2012;7:e37465. doi:10.1371/journal.pone.0037465

25 Muscogiuri G, Altieri B, de Angelis C, et al. Shedding new light on female fertility: The role of vitamin D. Rev Endocr Metab Disord 2017;18:273-83. doi:10.1007/s11154-017-9407-2

26 Chu J, Gallos I, Tobias A, Tan B, Eapen A, Coomarasamy A. Vitamin D and assisted reproductive treatment outcome: a systematic review and meta-analysis. Hum Reprod 2018;33:65-80. doi:10.1093/ humrep/dex326

27 Burgess S. Sample size and power calculations in Mendelian randomization with a single instrumental variable and a binary outcome. Int Epidemiol 2014;43:922-9. doi:10.1093/ije/dyu005

28 van Weert B, van den Berg D, Hrudey El, Oostvogels AJJM, de Mirand E, Vrijkotte TGM. Is first trimester vitamin D status in nulliparous women associated with pregnancy related hypertensive disorders? Midwifery 2016;34:117-22. doi:10.1016/j.midw.2015.12.007

29 Burris HH, Rifas-Shiman SL, Huh SY, et al. Vitamin D status and hypertensive disorders in pregnancy. Ann Epidemiol 2014;24:399403.e1. doi:10.1016/j.annepidem.2014.02.001

30 Powe CE, Seely EW, Rana S, et al. First trimester vitamin D, vitamin D binding protein, and subsequent preeclampsia. Hypertension 2010;56:758-63. doi:10.1161/ HYPERTENSIONAHA.110.158238

31 Adela R, Borkar RM, Mishra N, et al. Lower serum vitamin D metabolite levels in relation to circulating cytokines/chemokines and metabolic hormones in pregnant women with hypertensive disorders. Front Immunol 2017;8:273. doi:10.3389/fimmu.2017.00273

32 Behjat Sasan S, Zandvakili F, Soufizadeh N, Baybordi E. The effects of vitamin d supplement on prevention of recurrence of preeclampsia in pregnant women with a history of preeclampsia. Obstet Gynecol Int 2017;2017:8249264. doi:10.1155/2017/8249264

33 Hossain N, Kanani FH, Ramzan S, et al. Obstetric and neonatal outcomes of maternal vitamin D supplementation: results of an open-label, randomized controlled trial of antenatal vitamin D supplementation in Pakistani women. J Clin Endocrinol Metab 2014:99:2448-55. doi:10.1210/jc.2013-3491

34 Mojibian M, Soheilykhah S, Fallah Zadeh MA, Jannati Moghadam $M$. The effects of vitamin D supplementation on maternal and neonatal outcome: A randomized clinical trial. Iran / Reprod Med 2015;13:687-96.

35 Burgess S, Butterworth A, Malarstig A, Thompson SG. Use of Mendelian randomisation to assess potential benefit of clinical intervention. BMJ 2012;345:e7325. doi:10.1136/bmj. e732510.1136/bmj.e7325

36 Chaiworapongsa T, Chaemsaithong P, Yeo L, Romero R. Pre-eclampsia part 1: current understanding of its pathophysiology. Nat Rev Nephrol 2014;10:466-80. doi:10.1038/nrneph.2014.102

37 De-Regil LM, Palacios C, Lombardo LK, Peña-Rosas JP. Vitamin D supplementation for women during pregnancy. Cochrane Database Syst Rev 2016:1:CD008873 doi:10.1002/14651858.CD008873.pub3

38 US Institute of Medicine. Dietary reference intakes for calcium and vitamin D. Academy Press, 2010.

39 UK National Health Service. Vitamins, supplements and nutrition in pregnancy. 2015. https://www.nhs.uk/conditions/pregnancy-andbaby/pages/vitamins-minerals-supplements-pregnant.aspx.

40 Norwegian Health Directorate. Kostholds for gravide/Diet during pregnancy. 2018. https://helsedirektoratet.no/folkehelse/graviditetfodsel-og-barsel/graviditet-og-svangerskap/kosthold-for-gravide

41 World Health Organization. WHO recommendations on antenatal care for a positive pregnancy experience. 2016. http://www.who.int/ nutrition/publications/guidelines/antenatalcare-pregnancy-positiveexperience/en/

Web appendix: Supplementary material 